\title{
Corruption et fiscalité abusive internationales - Des pratiques onéreuses pour les entreprises ${ }^{1}$
}

\author{
Benoît Lapointe ${ }^{\mathrm{a}}$
}

RÉSUMÉ. Les conséquences de la corruption et des pratiques fiscales abusives internationales ne sont pas seulement négatives et coûteuses pour les États qui en subissent le fléau; elles le sont tout autant pour les entreprises qui en sont les auteurs, quoiqu'elles puissent prétendre le contraire. En réponse à cette situation de plus en plus insoutenable, les États s'organisent. Mais le combat ne sera gagné que si tous les pays et territoires coopèrent et harmonisent leurs actions et stratégies. Les entreprises n'auront alors d'autre choix que d'assumer leur responsabilité sociale en abandonnant les pratiques de corruption et de fiscalité abusives.

\begin{abstract}
The consequences of corruption and international abusive tax practices are not only negative and costly for the States that suffer their damaging effects; they are as much so for the firms engaging in them, even though they may assert the opposite. In response to this increasingly intolerable situation, the States get organized. But the fight will be of no avail if not all the countries and territories cooperate and harmonize their actions and strategies. Then the firms will have no option but to assume their corporate social responsability by renouncing their corruption and tax abusive practices.
\end{abstract}

Il peut être tentant, pour les gestionnaires d'entreprises, de se livrer à des pratiques de corruption ou de fiscalité abusive dans leurs activités internationales. Après tout, la théorie de l'agence n'enseignet-elle pas qu'en leur qualité d'agents de leurs principaux (propriétaires ou actionnaires), ils endossent la responsabilité de maximiser le chiffre d'affaires, les profits, et, conséquemment, l'augmentation de la valeur de l'entreprise que ces pratiques ont justement pour but de stimuler?

L'attrait n'est pourtant que de façade. Car les conséquences, ainsi que les coûts associés, de la corruption et des pratiques fiscales internationales abusives sont négatives, quel que soit l'angle d'approche envisagé. Les conventions internationales les énumèrent, tel un catalogue, dans leur préambule. Elles sont bien sûr néfastes pour les États au sein desquels elles sévissent, mais également pour les entreprises qui se livrent à ce genre de pratique, alors même qu'elles peuvent croire y trouver des avantages. Certaines, appuyées par une frange de la doctrine, prétendent pourtant le contraire afin de légitimer les pratiques qui en sont constitutives.

Les deux types de comportement, autant sur les plans juridique et politique, qu'économique et social, montrent d'ailleurs une ampleur et des corollaires analogues. Il s'agit en effet de pratiques dont les conséquences sont en quelque sorte "systémiques ", dans le sens où leurs répercussions, essentiellement négatives, dépassent leur propre cadre pour englober une multitude de dimensions étroitement reliées entre elles et qui conditionnent le fonctionnement harmonieux d'un État. Celui-ci est idéalement fondé sur le droit et l'équité, mais de tels comportements viennent marquer une rupture avec ces valeurs. Aussi convientil de les examiner en les associant, quoiqu'ils possèdent des caractéristiques propres.

Les travaux engagés par l'Organisation de coopération et de développement économiques (OCDE) à leur sujet, pour lesquels aujourd'hui le G20 apporte sa

a Chargé de cours, Université du Québec à Chicoutimi 
contribution ou sa caution, l'ont d'ailleurs été justement parce que les deux types de comportement sont préjudiciables autant pour les États qui les connaissent que pour les entreprises qui s'y livrent. Il convient de souligner, à cet égard, que, lorsqu'elles revêtent une dimension internationale, les pratiques de corruption ne sont pas vraiment éloignées de celles de fiscalité abusive (ou agressive) : elles sont proches parentes, même si, au premier regard, elles paraissent ne pas avoir de lien. Suivant le rapport de l'OCDE sur la corruption transnationale publié en 2014 , la deuxième grande catégorie d'« intermédiaires » intervenant dans les transactions internationales, qui ont joué un rôle dans $35 \%$ des 427 affaires qu'elle a répertoriées, est justement celle des "véhicules juridiques » (corporate vehicles), qui recouvrent, entre autres, des filiales et établissements implantés « dans des centres financiers ou des paradis fiscaux extraterritoriaux » (offshore $)^{2}$.

Il convient d'abord d'examiner sommairement les effets négatifs de la corruption sur les entreprises qui y ont recours (1), avant d'exposer les justifications que celles-ci, malgré tout, invoquent (2). Les pratiques fiscales internationales abusives peuvent ensuite être évoquées (3), en soulignant ensuite que si les entreprises n'adoptent pas spontanément un comportement socialement responsable à l'égard de ces pratiques, les États dont elles relèvent les obligeront à s'y soumettre. Cette responsabilisation, concrétisée essentiellement par des obligations de transparence, a d'ailleurs déjà commencé (4).

\section{Les effets néfastes de la corruption}

Les effets négatifs de la corruption sur les entreprises corruptrices elles-mêmes sont souvent comparés à ceux engendrés par l'imposition de taxes. À la différence de celles-ci, toutefois, puisque les sommes versées à l'agent public ne vont pas garnir le Trésor public, l'État se voit ainsi privé de ressources devant servir à l'amélioration du bien public, et, plus spécifiquement, des services et équipements publics. Des recherches ont même tenté de quantifier la magnitude des effets de la corruption sur la croissance d'une entreprise ainsi que sur sa performance économique (et financière). Ainsi, Raymond Fisman et Jakob Svennson ont estimé, dans l'une de leurs études, qu'un point de pourcentage d'augmentation du taux de corruption (défini par le montant des pots-de-vin divisé par celui du chiffre d'affaires) correspond à une diminution de plus de $3 \%$ du taux de croissance de l'entreprise. En conséquence, selon leurs calculs, l'impact négatif de la corruption sur la performance économique de l'entreprise est trois fois plus important que celui de la taxation (ou imposition ${ }^{3}$ ).

Mais la corruption entraîne des effets encore plus défavorables pour les entreprises qui s'y livrent, en raison, notamment, du haut niveau de secret dont elle est entourée, ainsi que de ses conséquences juridiques pour le moins aléatoires. Sur ce dernier plan, en effet, les entreprises ne peuvent faire appel aux tribunaux, nationaux ou arbitraux, pour obtenir l'exécution des actes devant être accomplis par l'agent public ou privé si celuici fait défaut de s'exécuter ou le fait inadéquatement, sans respecter la lettre ou l'esprit du "pacte de corruption » intervenu. Privées d'exécution en nature, elles ne peuvent non plus obtenir un dédommagement sous forme de dommagesintérêts. D'habitude secrète, l'existence des " commissions » versées apparaît alors en pleine lumière lorsqu'elles sont au centre de litiges dont les tribunaux peuvent être appelés à connaître, et plus spécialement les tribunaux arbitraux, qui agissent fréquemment en tant qu'organes juridictionnels en matière commerciale.

D’une manière générale, les diverses (et encore rares à ce jour) affaires de corruption portées à titre principal et en tant que telles devant des juridictions arbitrales ont donné lieu à une condamnation pure et simple de cette pratique. Cette réprobation s'est exprimée de deux manières différentes et successives, mais qui marquent toutes deux la volonté des tribunaux arbitraux de refuser de donner leur aval aux pratiques de corruption dans le cadre des transactions commerciales internationales.

Une première démarche, procédant sans doute de la tradition anglo-américaine, s'est exprimée dans une sentence très remarquée, rendue en 1963 par l'arbitre (et également juge) Gunnar Lagergren ${ }^{4}$. Elle concernait un litige relatif à un contrat conclu entre une société britannique et un intermédiaire argentin auquel une commission de $10 \%$ de la valeur du contrat avait été consentie. L'arbitre a conclu que les contrats qui violent sérieusement les bonnes mœurs ou l'ordre public international sont non valables, 
que ce soit devant des juridictions nationales ou arbitrales; ils ne peuvent, à tout le moins, donner lieu à un recours en exécution forcée. En l'espèce, après avoir relevé que la commission réclamée par le demandeur avait pour destination probable le versement de pots-de-vin, il déclina sa compétence au nom de ce principe général de droit. Les parties qui s'allient dans une telle entreprise, ajoute l'arbitre, doivent réaliser qu'elles ont perdu tout droit de s'adresser à la justice afin de recevoir sa sanction, que les litiges soient portés devant des juridictions nationales ou des tribunaux arbitraux.

Cette décision demeure tout de même paradoxale dans la mesure où, après que l'arbitre a constaté l'illicéité du contrat de commission, il n'en a pas tiré les conséquences en prononçant sa nullité. C'est pourquoi une telle approche est aujourd'hui délaissée par les juridictions arbitrales, qui ne refusent plus de connaître du fond d'un litige même si le recours en exécution porté devant elles se fonde sur un accord qui viole l'ordre public ou les bonnes mœurs, en l'occurrence par des pratiques de corruption. Aussi les sentences rendues ces dernières années dans de telles circonstances se sont-elles déplacées sur un autre terrain : les tribunaux arbitraux, n'éludant pas le débat de fond, elles ont rejeté des demandes en paiement de " commissions", prononçant la nullité des engagements invoqués au motif de l'illicéité des dispositions contractuelles s'y rapportant.

Par exemple, un tribunal arbitral, dans une telle situation, a affirmé sa compétence sur la base de l'autonomie de la clause compromissoire ${ }^{5}$, cette dernière n'étant pas affectée par la nullité frappant les autres stipulations du contrat dont elle faisait par ailleurs partie ${ }^{6}$. Il y a été prononcé qu'une convention par laquelle une personne s'engage, moyennant contrepartie, à user de son influence auprès d'une administration publique afin d'obtenir un marché au bénéfice de son cocontractant, est contraire aux bonnes mœurs ainsi qu'à l'ordre public (interne), et aussi, en dehors de toute loi nationale, «à ce qui est considéré être la moralité dans les affaires internationales ».

Il en va pareillement d'une autre affaire dans laquelle un tribunal arbitral eut à statuer sur un différend relatif au versement d'une commission par une entreprise française à une entreprise britannique dont elle avait sollicité le concours pour l'obtention de marchés à conclure avec le gouvernement d'un État d'Afrique. Se fondant sur le fait que la commission convenue devait servir en réalité à verser des pots-de-vin aux responsables locaux, le tribunal estima que la cause (sur le plan juridique) de l'engagement souscrit par l'entreprise française à l'égard de l'intermédiaire britannique était illicite et immorale au regard autant de l'ordre public français interne qu'en vertu de la conception de l'ordre public international tel que la plupart des nations le reconnaissent. L'accord conclu devenait dès lors nul et de nul effet, ce qui interdisait aux parties de s'en prévaloir?

Pour sa part, la Cour d'appel de Paris a expressément reconnu le pouvoir de l'arbitre de refuser de rendre exécutoires, et même celui de déclarer nuls, des contrats ayant pour objet le versement de commissions qui, à leur base, sont illicites ${ }^{8}$. Il s'agissait, en l'espèce, d'un recours en annulation d'une sentence pour contrariété à l'ordre public français et à l'« ordre public réellement (ou véritablement) international », en ce sens que son exécution en France aurait eu pour conséquence de donner effet à un contrat illicite par sa cause et son objet, qui consistaient, en l'occurrence, en l'exercice d'un trafic d'influence par le versement de pots-de-vin. Quoique le tribunal ait rejeté le moyen tiré de la nullité de la sentence pour contrariété à l'ordre public international parce que la preuve n'avait pas été faite de l'illicéité du contrat pour trafic d'influence ou corruption, il formula, chose curieuse en droit français, une sorte d'obiter dictum $^{9}$, suivant lequel « $[\ldots]$ un contrat ayant pour cause ou pour objet l'exercice d'un trafic d'influence par le versement de pots-de-vin est, en conséquence, contraire à l'ordre public international français ainsi qu'à l'éthique des affaires internationales telle que conçue par la plus grande partie des États de la communauté internationale. »

À la lumière de ces quelques sentences ${ }^{10}$ et de l'arrêt de la Cour d'appel de Paris, on constate que le problème de la corruption dans les transactions commerciales internationales, lorsqu'il surgit à l'occasion de différends portés devant des tribunaux judiciaires ou arbitraux, est appréhendé par le droit à travers la notion d'« ordre public » : l'illicéité de telles pratiques est affirmée 
par référence aux exigences de l'ordre public du droit national gouvernant le contrat litigieux, ou même à celles d'un ordre public « réellement international ", notion controversée, expression d'un principe général de droit reconnu par la plupart des États.

Comme les «pactes de corruption » ne peuvent être exécutés juridiquement, et qu'en outre ils comportent des conséquences négatives pour les entreprises qui les concluent, pourquoi, dès lors, sont-ils si répandus? Les entreprises qui ont recours à ce genre de pratique avancent, en ce sens, des justifications.

\section{La corruption indûment légitimée}

Une entreprise peut consacrer beaucoup de temps à « rechercher » un agent potentiellement corrompu, puis à négocier avec lui une entente secrète, autant de temps qu'elle ne consacre pas, en particulier, à l'amélioration de ses structures, à l'innovation ou au caractère concurrentiel de son activité et de ses produits ou services. Ne serait-ce que pour cette raison, une entreprise devrait éviter de se livrer à ce type de comportement.

Cependant, en dépit des nombreux effets négatifs sur les entreprises qui décident d'entrer dans le jeu de la corruption, certaines, parmi celles originaires des États développés, plaident pourtant que la corruption est un mal nécessaire, si l'on considère que les pratiques qui la constituent sont intrinsèques à la culture de certains États, en particulier en développement; ou encore, que la corruption est comparable à une sorte de taxe informelle à laquelle elles ne peuvent se soustraire si elles veulent exercer une activité au sein de ces États.

Les entreprises qui défendent un tel point de vue ne sont pas seules dans leur croisade visant à faire accepter les pratiques de corruption. Bien que l'on peine à identifier des partisans de la corruption sur le seul plan de l'éthique, quelques auteurs font néanmoins encore valoir qu'elle peut servir des fins économiques louables. Selon eux, devant une bureaucratie inepte ou un appareil gouvernemental inefficient, la corruption peut augmenter le bienêtre social général de la population de l'État où elle sévit. Si les ressources de l'État ne sont pas allouées de façon efficiente en raison de ces dysfonctionnements de l'appareil public, la corruption peut réin- troduire une sorte de mécanisme de marché qui assure une meilleure (ou moins mauvaise) répartition des ressources, quoique de manière différente, c'est-à-dire en fonction de la "volonté de payer » des entreprises. La corruption permet l'introduction d'un élément de concurrence, et donc d'efficience économique, dans ce qui est normalement un système de monopole. Il s'agit là de ce que l'on a coutume de nommer " l'huile ou la graisse (lubrifiant) dans les rouages du commerce ou des affaires " (oil or grease in the wheels of commerce or business $\left.{ }^{11}\right)$. Nathaniel $H$. Leff et Samuel P. Huntington, notamment, sont bien connus pour leur plaidoyer en faveur d'une telle conception.

Les tenants de ce point de vue reconnaissent toutefois qu'il est difficile de borner les bienfaits de la corruption au seul accroissement de l'efficience économique au sein d'un État. Ils mentionnent, à cet égard, une panoplie de conséquences négatives associées à la corruption, comme une réallocation des ressources vers les riches et les détenteurs du pouvoir politique, ou encore le cynisme de la population envers la classe politique et les institutions étatiques. Une vision alternative est proposée par ceux qui, en raison de ces conséquences calamiteuses, limitent le caractère acceptable de la corruption aux paiements de facilitation, à savoir le versement de sommes ou l'attribution d'autres avantages à des agents publics étrangers afin qu'ils accomplissent - ou le fassent plus rapidement leurs fonctions habituelles, comme le dédouanement de marchandises. Pourtant, la corruption peut comporter des effets corrosifs même à cette moindre échelle. Elle semble de prime abord sans conséquence, mais la corruption au moyen de paiements de facilitation versés à des agents publics qui sont situés en bas de la hiérarchie de l'appareil bureaucratique peut contribuer à une culture de non-droit, propice à la génération et au développement d'activités bien plus graves, comme le crime organisé.

Quoique certains fassent valoir que, spécialement dans le cas des paiements de facilitation, les versements effectués ne sont rien de plus que l'huile ou le lubrifiant nécessaire pour exercer une activité économique dans un environnement qui n'y est généralement pas favorable, il faut reconnaitre que la corruption ne se limite généralement pas à ce genre de situation. S'ils ne sont pas vigoureusement combattus, les paiements de facilitation peuvent, en 
créant une culture de la corruption, dégénérer, par un effet de spirale, en de véritables versements de pots-de-vin. Même quand les paiements de facilitation ou les pots-de-vin se révèlent (de façon temporaire, cela s'entend) positifs sur le plan économique, les gouvernements et les entreprises ne devraient pas les tolérer. Ils devraient plutôt agir vigoureusement afin d'éviter que ne s'instaure une culture de l'illégalité. Car les marchés illégaux sont toujours inefficients sur le plan économique et, plus généralement, inéquitables, lorsqu'on les compare aux marchés légaux. Aussi comprend-on pourquoi l'OCDE recommande instamment à ses États membres de proscrire leur utilisation par les entreprises qui détiennent leur nationalité ou qui, autrement, entrent dans leur champ de compétences personnel. C'est ce qu'a d'ailleurs fait le Canada lorsqu'il a modifié, en 2013, sa Loi sur la corruption d'agents publics étrangers, quoiqu'il a pris quatre ans afin de mettre en vigueur, le 31 octobre 2017, la disposition correspondante ${ }^{12}$.

Derrière la condamnation de la corruption, l'on retrouve en vérité, par-delà les arguments moraux, une approche anglo-saxonne, empreinte de libéralisme : le bon fonctionnement des règles du marché est garant du bien public. C'est grâce à la concurrence juste et transparente sur le marché que les consommateurs accèdent à un plus grand éventail de biens et services et ce, aux meilleurs prix. Dans cette logique, la corruption, en faussant le jeu de la concurrence, nuit au consommateur et, par là même, au bien public général. Il est, par conséquent, du devoir de l'État, responsable du bon fonctionnement du marché, et donc $\mathrm{du}$ respect d'une saine concurrence, de combattre la corruption.

Même si, en soi, un acte isolé de corruption de la part d'une entreprise peut se révéler efficient sur le plan économique, il contribue néanmoins à l'acceptation de la pratique de la corruption au sein du marché et de la société en général. Un tel comportement est dommageable, à moyen et long termes. Il ne profite - de façon provisoire il va sans dire qu'à ceux qui s'y livrent; tous les autres en souffrent. Le dommage, insidieux, vient surtout de l'effritement qu'il provoque à l'égard du respect de la règle de droit (rule of law) et des éléments constitutifs de l'État de droit. Cette conséquence est dévastatrice sur tous les plans; elle condamne sans ap- pel le développement économique, social, institutionnel et juridique des États au sein desquels la corruption s'enracine.

La corruption, en gangrenant l'État, n'est pas un phénomène dont celui-ci peut s'extirper naturellement sans une réforme institutionnelle et de sa gouvernance. Chaque État doit accomplir un effort individuel. En cela, il peut recevoir l'aide de nombreux partenaires. Ainsi, autant le Fonds monétaire international (FMI), la Banque mondiale que l'OCDE, à l'unisson d'un nombre accru d'organisations non gouvernementales (ONG), ont engagé une action en faveur de la lutte contre la corruption dans le monde. La conjugaison de leurs démarches et de celles de leurs États membres n'est pourtant pas suffisante. Les entreprises elles-mêmes doivent agir. Comptant parmi les parties prenantes principales (stakeholders) dans la gouvernance d'un État, elles forment un élément essentiel de sa structure économique et sociale. Leur influence et leur pouvoir, loin de se limiter au domaine des affaires, s'étendent et s'exercent sur la société entière. Elles ont donc le devoir d'œuvrer au maintien et au raffermissement de l'environnement juridique et concurrentiel dans lequel elles exercent leur activité si elles veulent être en mesure de bénéficier des avantages résultant de l'application des « règles du jeu » normales de la concurrence. Les entreprises, personnes juridiques (morales) qui ne doivent leur naissance qu'aux systèmes juridiques nationaux qui les ont enfantées, ont ainsi une obligation morale de s'abstenir des pratiques de corruption.

Plusieurs gouvernements d'États en développement ont d'ailleurs réalisé qu'il y allait de leur propre intérêt économique d'établir des conditions de concurrence égales pour tous, et donc de lutter contre les pratiques de corruption. Il faut d'ailleurs préciser que la pression est aussi venue des États donateurs, des ONG, et en particulier de Transparency International. Plus tard, la Banque mondiale a ajouté sa voix, ainsi que le FMI. Le président James D. Wolfensohn, dans une décision historique de 1996, plaça en effet la corruption parmi les priorités de la Banque. En somme, une prise de conscience des maux de la corruption, et de la nécessité conséquente de l'attaquer, a progressivement émergé. Il en va de même à propos des pratiques de fiscalité internationale abusives. 


\section{Les pratiques fiscales internationales abusives}

L'utilisation des États ou territoires ${ }^{13}$ à fiscalité privilégiée (paradis fiscaux classiques et partiels), voire non coopératifs (ETNC), c'est-à-dire qui refusent l'échange de renseignements fiscaux et financiers avec les autres États ou territoires, suscite des interrogations qui tiennent à l'éthique des affaires, et, plus largement, à la responsabilité sociale - ou sociétale - de l'entreprise (RSE) $)^{14}$. Car, même si l'utilisation de ces États ou territoires est encore permise aujourd'hui dans de nombreux cas, quoique bien souvent en marge de la législation nationale et des conventions fiscales, qu'en est-il pourtant sur un plan éthique? Comment ces pratiques, dont certaines, à la limite de la légalité, peuvent ne pas être abusives en tant que telles et ainsi faire l'objet de sanctions (pour l'instant du moins), se justifient-elles dans l'optique de la RSE?

En effet, par-delà le traitement juridique de la question, c'est aussi à ce niveau que se situe aujourd'hui le débat qui se déroule à propos des pratiques fiscales « douteuses » des groupes internationaux de sociétés, en particulier les plus grands d'entre eux. C'est ainsi qu'un rapport publié le 3 décembre 2012 par la commission des finances publiques de la Chambre des communes du Royaume-Uni a qualifié les pratiques fiscales agressives (ou abusives) des sociétés américaines comme Google, Apple, Amazon, Starbucks, comme étant «immorales ». Certains considèrent également qu'elles constituent une « ingénierie, contraire à l'éthique, [qui] offre une échappatoire aux règles fondamentales selon lesquelles l'impôt est un devoir et l'évasion fiscale une pratique légale [sic] mais immorale ${ }^{15}$. » Ed Miliband, l'ex-leader du Parti travailliste britannique, pour sa part, dans une allocution prononcée le 22 mai 2013, a parlé, à propos des pratiques fiscales de ces mêmes sociétés du secteur des technologies de l'information et de la communication (TIC), du « capitalisme responsable » (responsible capitalism) dont les «sociétés responsables » (responsible companies) doivent faire preuve.

La mobilisation actuelle contre les paradis fiscaux semble à cet égard bénéficier de celle qui s'est formée autour de la RSE. Plus largement, les questions fiscales, ces dernières années, en sont venues à dépasser la seule sphère juridique aux yeux du grand public certes, mais aussi des parties prenantes de l'entreprise, comme les clients et fournisseurs, et des dirigeants eux-mêmes, pour revêtir une dimension éthique et sociale. Aussi les entreprises sont aux prises aujourd'hui avec un risque sérieux, qu'elles reconnaissent volontiers, de mauvaise réputation, de " déficit d'image ", qui résulte de leur utilisation des États ou territoires à la fiscalité privilégiée, et, plus généralement, de leurs pratiques fiscales douteuses, en particulier lorsque celles-ci sont révélées au public par les ONG.

Toute la problématique revient alors à identifier ce qui est acceptable et ce qui ne l'est pas. En la matière, la réponse des dirigeants d'entreprise, même si la responsabilité sociale s'inscrit désormais dans leur réalité, est pourtant différente de celle des pouvoirs publics ${ }^{16}$. Les premiers prétendent que leur utilisation des régimes fiscaux privilégiés est due en grande partie à la nécessité économique de faire baisser les coûts, y compris fiscaux, jusqu'à un niveau acceptable. Ils mettent l'accent, en ce sens, sur les contradictions existant entre les législations nationales ou sur l'absence de coordination entre elles, génératrices, à leur avis, de surimpositions pour les entreprises qui réalisent des opérations internationales. Les dirigeants d'entreprise (agents) estiment à cet égard avoir envers leurs actionnaires (principaux) le devoir de réduire, par des moyens licites, les impôts dont sont redevables les sociétés dont ils assurent la gestion. Ils font, en réalité, allusion à leur devoir, en tant qu'agents, de réduire les coûts qui échoient à leurs principaux, suivant la théorie de l'agence. Ils jugent par conséquent infondées la plupart des accusations les visant à propos de leurs pratiques fiscales hasardeuses, tenant même les pouvoirs publics pour responsables de politiques fiscales incohérentes et de systèmes fiscaux qui incitent à l'érosion des bases d'imposition et au transfert de bénéfices dans les États ou territoires à l'imposition favorable. Selon eux, il faut aussi considérer que les procédures amiables que comportent les conventions fiscales, souvent longues (en général, quatre ans), ne parviennent pas toujours à résoudre en temps voulu les différends entre États, d'autant qu'en dehors de l'Union européenne (UE -et à propos seulement des litiges portant sur les prix de transfert), les administrations fiscales ne sont pas tenues de les régler.

Les seconds, de leur côté, mettent l'accent sur le fait que l'existence de tels régimes fiscaux privilégiés est un facteur déterminant d'orientation des capitaux en fonction de motivations de nature fiscale. Ces régimes portent ainsi atteinte au principe de la neutralité de l'impôt, qui vise à ce que les systèmes fiscaux nationaux n'incitent ni à l'exportation ni à l'importation de 
capitaux. Ils faussent, de surcroit, les conditions de la concurrence, dès lors qu'en pratique seuls certains contribuables, en général les plus puissants, peuvent les utiliser. Les pouvoirs publics sont également sensibles aux pertes de recettes fiscales qui proviennent d'une érosion des bases d'imposition des entreprises résidentes. Car l'argent dont ils sont ainsi privés ne peut servir à la fourniture des services publics et à l'amélioration des infrastructures qui leur servent de support, qu'utilisent par ailleurs les entreprises mêmes qui se soustraient à l'impôt.

Le tracé de la ligne de partage entre ces positions opposées ne peut pourtant être effectué en se fondant sur la science juridique, car l'efficacité de la règle de droit atteint ici ses limites. C'est plutôt en se plaçant dans la perspective de la RSE que l'on peut tenter d'y parvenir ${ }^{17}$.

Cette voie, a priori porteuse d'espoir, est cependant décevante elle aussi, du moins pour l'instant; une grande partie de la solution à cette problématique y réside pourtant, à moyen et long termes. Car, malgré tout le développement que la RSE a connu ces dernières décennies, l'on s'étonne que la question du comportement fiscal " socialement acceptable », en particulier lorsqu'il comporte une dimension internationale, n’y figure que de façon incidente, voire négligeable, alors que l'on serait d'emblée porté à croire que la fiscalité compte parmi ses champs prioritaires de couverture. Le constat actuel d'impuissance de la RSE devant l'indiscipline fiscale des groupes internationaux de sociétés apparaît ainsi paradoxal.

Cet état de choses est toutefois en train de se modifier, ne serait-ce que de façon très graduelle. La doctrine donne le ton : de plus en plus d'ouvrages et de colloques sont consacrés à la question. C'est sans doute le signal que la situation évolue. Parce qu'il ne peut en être autrement : la RSE ne peut méconnaitre le pan essentiel que constitue le comportement fiscal responsable, tout comme les entreprises ne sauraient, de leur côté, faire fi de la RSE.

Le chemin à parcourir demeure cependant encore long et semé d'embûches. En témoigne l'examen de la nouvelle norme $\mathrm{ISO}^{18} 26000$, premier pas en ce sens quoique timide. Cette frilosité est d'ailleurs favorisée par les conclusions de certaines études. Celles-ci démontrent en effet que les pratiques fiscales douteuses des sociétés n'ont que peu d'impact en réalité sur leur réputation globale et l'image qu'elles projettent sur leurs parties prenantes, de même que sur leurs résultats financiers. Un tel constat relativise le risque de déficit d'image et de mauvaise réputation qui a été évoqué plus haut.

La RSE étant, en l'état, pour le moins embryonnaire sur la question des pratiques fiscales internationales abusives, il est à se demander d'où peut provenir, en attendant son évolution future, le signal d'un changement d'attitude de la part des entreprises? Probablement d'une évolution des pratiques des conseillers fiscaux et d'un durcissement des règles déontologiques applicables à ces fameux « intermédiaires » ou « promoteurs » dont parle tant l'OCDE et qui sont les instigateurs imaginatifs des pratiques fiscales internationales abusives. Il émanera au premier chef de ceux qui sont membres d'un ordre professionnel. Mieux vaut que cela se fasse rapidement. Car les législateurs sont déjà à la tâche.

L'OCDE, en tout cas, pousse fortement à la roue, incitant avec vigueur et sagesse les entreprises à agir, par crainte, tout spécialement, de la réalisation de ce « risque fiscal» tant redouté. Aussi Les principes directeurs de l'OCDE à l'intention des entreprises multinationales, dans leur version de 2011, consacrent-ils un chapitre entier (XI) à la fiscalitéé ${ }^{19}$. L'OCDE indique en effet, au paragraphe 2, que « [l]es entreprises devraient considérer la gouvernance fiscale et la discipline fiscale comme des éléments importants de leurs mécanismes de contrôle et de leurs systèmes de gestion des risques au sens large. En particulier, les conseils d'administration devraient adopter des stratégies de gestion du risque fiscal qui permettent d'identifier et d'évaluer pleinement les risques financiers, réglementaires et de réputation associés à la fiscalité». Le commentaire (no 102) portant sur ce principe mentionne la responsabilité qui échoit sur ce plan aux mandataires sociaux (dirigeants et membres des conseils d'administration):

Les engagements des entreprises en matière de coopération, de transparence et de discipline fiscale devraient se refléter dans des systèmes, structures et politiques de gestion des risques. Pour les entreprises constituées en société, les conseils d'administration sont en mesure de contrôler le risque fiscal par divers moyens. Par exemple, le conseil d'administration devrait, de manière anticipative, élaborer des principes appropriés de politique fiscale, et mettre en place des systèmes de contrôle interne garantissant que les actions des dirigeants sont conformes à l'analyse du risque 
fiscal effectuée par le conseil. Le conseil devrait être tenu informé de tout risque fiscal potentiellement grave, et les responsabilités devraient être établies pour l'exercice des fonctions internes de contrôle fiscal et de communication d'informations au conseil. Une stratégie globale de gestion des risques comportant un volet fiscal permettra à l'entreprise non seulement de faire preuve de civisme, mais aussi de gérer efficacement le risque fiscal, lui permettant ainsi de se prémunir contre d'importants risques financiers, réglementaires et de réputation.

On ne saurait mieux dire. Les principes directeurs de l'OCDE s'inscrivent résolument dans une optique de RSE. Le langage employé en est d'ailleurs révélateur, les principes et les commentaires associés utilisant notamment les expressions de " civisme » et de " conduite responsable » des entreprises en regard de leurs pratiques fiscales. En ce sens, les principes soulignent, au paragraphe 1, que " les entreprises devraient se conformer à la lettre comme à l'esprit des lois et règlements fiscaux des pays où elles opèrent. Respecter l'esprit de la loi signifie comprendre et suivre l'intention du législateur ». Comment ne pas y reconnaitre l'abus de droit? Suivant le commentaire (no 100) de l'OCDE à propos de ce dernier principe :

$[u]$ ne entreprise respecte l'esprit des lois et règlements fiscaux si elle prend des mesures raisonnables pour déterminer l'intention du législateur et interprète ces règles fiscales conformément à cette intention, à la lumière du texte de loi et de la jurisprudence contemporaine pertinente. Les transactions ne devraient pas être structurées en vue d'obtenir des résultats fiscaux sans rapport avec les conséquences économiques sous-jacentes de la transaction, sauf s'il existe une législation spécifique destinée à produire ce résultat. En pareil cas, l'entreprise devrait avoir de bonnes raisons de penser que la transaction est structurée de telle sorte qu'elle aboutit à un résultat fiscal qui n'est pas contraire à l'intention du législateur.

De tels propos comportent une nette exhortation aux entreprises de ne pas s'adonner à la planification fiscale agressive, que l'OCDE considère comme contraire à un comportement fiscal responsable.
Quoi qu'il en soit, l'appel à agir, s'il n'est pas suivi d'effet, c'est-à-dire par l'adoption spontanée et généralisée d'un comportement responsable sur le plan fiscal de la part des entreprises, se transformera en obligation législative. Aussi voit-on poindre les conséquences potentielles, sur le plan juridique, pour les entreprises, leurs mandataires sociaux et leurs actionnaires, d'un comportement fiscal non responsable : l'intervention du législateur. La RSE changera alors de nature - d'extrajuridique qu'elle était, le demeure encore en grande partie et devrait continuer de l'être -, pour devenir progressivement une contrainte imposée par le droit positif. La métamorphose a d'ailleurs déjà commencé vu l'inertie constatée des entreprises en ce qui concerne la modification de leurs pratiques fiscales internationales.

\section{L'imposition d'un comportement fiscal responsable}

L'imposition, par la loi, d'un comportement fiscal responsable est la voie que suggère d'emprunter le rapport déposé par le Sénat de France en juillet 2012. Sa proposition no 6 recommande en effet d'« [i]nclure le civisme fiscal des entreprises dans le champ de leur responsabilité sociale ${ }^{20} »$. La commission des Finances, de l'Économie générale et du Contrôle budgétaire de l'Assemblée nationale française a adopté une position identique dans le rapport qu'elle a déposé le 10 juillet 2013. Dans sa proposition no 20, elle recommande d'« [é]largir le champ de la responsabilité sociétale et environnementale des entreprises aux conséquences fiscales de leurs activités et de leurs stratégies. »Car, à son avis, le civisme fiscal fait partie de la RSE. Son point de vue se fonde en particulier sur le fait que l'optimisation fiscale à laquelle procèdent les entreprises se traduit souvent par des choix de localisation des actifs, qu'ils soient physiques ou immatériels, qui entraînent des implications très concrètes sur le plan de l'emploi. En outre, la cohésion sociale est notamment assurée par le financement, au moyen de l'impôt, de services et d'équipements publics. En ce sens, l'impôt a pour vocation de financer des dépenses publiques dont tous bénéficient, les entreprises tout autant que les citoyens. Le comportement fiscal d'une entreprise a donc bien des conséquences sociales, et il serait légitime, selon la commission des Finances, de l'Économie générale et du Contrôle budgétaire, de l'inclure officiellement dans le champ de la $\mathrm{RSE}^{21}$. La question demeure pourtant de savoir quelle forme cette RSE contrainte doit revêtir. 
On peut cependant déjà en voir les prémices. Car si les entreprises n'agissent pas volontairement en ce sens, l'extension, par les pouvoirs publics, de la RSE de type normatif à leur comportement fiscal va se traduire par l'instauration d'une publicité obligatoire de leurs agissements fiscaux. Celle-ci s'ajoutera, en France, à l'obligation de divulgation du bilan environnemental et social des sociétés cotées instaurée par la loi relative aux nouvelles régulations économiques ${ }^{22}$, qui a été étendue à certaines sociétés non cotées par la loi dite « Grenelle $\mathrm{II}^{23}$ ». Les informations fournies doivent faire l'objet d'une vérification par un organisme tiers indépendant, contrôle qui donne lieu à un avis qui est transmis à l'assemblée des actionnaires ou des associés en même temps que le rapport du conseil d'administration ou du directoire devant les contenir.

Ces contraintes accrues de divulgation entraîneront une responsabilité plus grande de la part des mandataires sociaux. En conséquence, les entreprises devront instituer en leur sein des procédures transparentes de décision et de prévention des risques fiscaux. Elles seront aussi tenues d'introduire des mécanismes permettant de contrôler le respect de ces procédures, de la même manière que pour assurer celui de l'obligation existant déjà, en France, en matière de transparence financière, depuis la loi no 2003-706 du $1{ }^{\text {er }}$ août 2003 de sécurité financière ${ }^{24}$.

L'exigence d'une transparence rehaussée de la part des entreprises est, par conséquent, déjà une réalité dans les systèmes juridiques de certains États. Appelée inéluctablement à s'amplifier à l'avenir et à entraîner dans sa course davantage d'États, son aboutissement ultime va consister, pour les entreprises exerçant leur activité au sein de plusieurs États ou territoires, à devoir rendre publiques, pour chaque État d'implantation, certaines données clés, dont, en particulier, le nombre d'établissements et de filiales, leurs effectifs, leur chiffre d'affaires, les bénéfices réalisés, les impôts acquittés ${ }^{25}$. En ce sens, les mesures introduites à l'endroit des établissements de crédit, compagnies financières et compagnies financières holding mixtes et entreprises d'investissement français, par la loi no 2013-672 du 26 juillet 2013 de séparation et de divulgation des activités bancaires, qui les contraint à divulguer toute une série d'informations relatives à leur activité dans les États ou territoires à fiscalité privilégiée ${ }^{26}$, ne représentent qu'un avant-goût de l'environnement législatif futur auquel les entreprises doivent s'attendre.
Plus récemment, à la fin de 2016, le Parlement français a adopté la loi dite "Sapin II », du nom du ministre qui l'a parrainée ${ }^{27}$. Transposant la directive européenne 2016/881 du Conseil du 25 mai 2016 dont il sera question plus bas, elle introduit une obligation de transparence à l'endroit des multinationales réalisant un chiffre d'affaires consolidé supérieur à 750 millions d'euros, et tout spécialement la publication d'informations détaillées sur les impôts dont elles s'acquittent dans les États ou territoires où elles exercent leur activité. Doivent ainsi être déclarés, dans un rapport annuel accessible gratuitement au public sur Internet, pour chacun des États membres de l'UE dans lesquels les sociétés concernées exercent leur activité, le nombre de salariés, le montant du chiffre d'affaires net, le montant du résultat avant impôt sur les bénéfices, le montant de l'impôt sur les bénéfices dû, le montant de l'impôt acquitté accompagné d'une explication sur les discordances éventuelles avec le montant dû, et le montant des bénéfices non distribués. D’autres obligations déclaratives sont prévues en ce qui a trait aux États situés en dehors de l'UE dans lesquels les sociétés en question exercent leur activité. Le Conseil constitutionnel a cependant déclaré la disposition législative qui institue cette obligation, soit le paragraphe I de l'article 137 de la loi, contraire à la Constitution. Par la même occasion, et pour les mêmes motifs, la Haute juridiction a déclaré inconstitutionnels les paragraphes III à V de l'article 7 de la loi du 26 juillet 2013 qui vient d'être évoquée, et qui avaient institué un dispositif semblable ${ }^{28}$.

Par ailleurs, la Commission européenne, dans le cadre de son "paquet de mesures sur la transparence fiscale » présenté le 18 mars 2015, avait elle aussi envisagé, entre autres, la possibilité de proposer des mesures de divulgation, État par État, des activités exercées, des bénéfices réalisés et des impôts payés, de la part de chacune des implantations des entreprises ayant des activités dans plusieurs États de l'UE. Les intentions en ce sens de la Commission européenne ont ensuite été précisées le 17 juin suivant, date du dévoilement de son «plan d'action pour une fiscalité des entreprises plus juste et plus efficace au sein de l'Union $»^{29}$.

Ainsi, la Commission européenne a-t-elle mis en chantier un certain nombre de réformes dans le but d'accroître la transparence fiscale des entreprises. Elle s'est d'abord appliquée aux aspects techniques d'un texte ayant pour but de réglementer les décisions fiscales anticipées abusives, dont l'affaire LuxLeaks, révélée à la fin de 2014, a pu 
montrer la prodigalité du Luxembourg à cet égard. L'obligation à laquelle les Vingt-Huit doivent désormais se conformer a été proposée par la Commission européenne à la fin 2014. Elle fut adoptée le 8 décembre $2015^{30}$ avec une célérité inhabituelle par l'ensemble des États membres, les plus réticents jusqu'alors à toute avancée en matière de lutte contre la fraude et l'évasion fiscales (Luxembourg, Pays-Bas, Belgique, Irlande) ayant dû céder sous la pression des opinions publiques. La directive 2011/16/UE du 15 février 2011 en matière de coopération administrative dans le domaine fiscal fut ainsi modifiée par l'introduction d'une obligation d'échange automatique et obligatoire entre les États membres de leurs décisions fiscales anticipées (rescrits fiscaux ou rulings) concernant les entreprises. La modification introduite a pour but de remédier à l'ignorance dans laquelle les États membres de l'UE étaient auparavant laissés s'agissant des décisions anticipées rendues par leurs voisins à propos, notamment, des prix de transfert, domaine de prédilection de ces décisions, mais qui peuvent aussi concerner, plus largement, la fiscalité d'ensemble de ces entreprises.

Ainsi, depuis le $1^{\text {er }}$ janvier 2017, les administrations des vingt-huit États membres de l'UE doivent se communiquer, sur une base automatique et obligatoire, tous les accords fiscaux préalables qu'elles concluent avec les entreprises sur un plan transfrontalier. Le but de cette transparence est d'éviter qu'un État membre n'octroie des conditions fiscales trop avantageuses à une multinationale et ne prive ainsi les autres États membres de substantiels revenus liés à l'activité réelle de cette société sur leur territoire. Le fait d'être mutuellement tenus au courant de tous les accords fiscaux préalables devrait permettre d'en finir avec cette forme de concurrence fiscale dommageable dans l'UE.

Le 28 janvier 2016, la Commission européenne a également proposé d'étendre les échanges d'informations aux déclarations pays par pays. Le 25 mai 2016, le Conseil des ministres de l'Économie et des Finances (ECOFIN) a, en ce sens, officiellement adopté une directive modifiant la directive 2011/16/UE en ce qui concerne l'échange automatique et obligatoire d'informations dans le domaine fiscal ${ }^{31}$. La directive impose en effet des exigences de transparence aux groupes d'entreprises multinationales de l'UE, ainsi qu'à l'égard de ceux hors de l'UE dont une ou plusieurs entités y ont leur siège.
Dans leur déclaration pays par pays, les groupes d'entreprises multinationales doivent ainsi fournir, chaque année et pour chaque juridiction fiscale dans laquelle ils exercent une activité, le montant de leur chiffre d'affaires, leur bénéfice avant impôts, les impôts sur les bénéfices qu'ils ont acquittés et ceux qui sont dus. Ils sont également tenus d'indiquer leur nombre d'employés, leur capital social, leurs bénéfices non distribués et leurs actifs corporels dans chaque juridiction fiscale. Ils sont enfin dans l'obligation d'identifier chacune des entités du groupe qui exerce une activité dans une juridiction fiscale donnée et, pour chacune d'elles, indiquer la nature de cette activité. Seuls les groupes d'entreprises multinationales dont le chiffre d'affaires consolidé annuel est égal ou supérieur à 750 millions d'euros seront obligés de remplir la déclaration pays par pays. Les déclarations seront échangées automatiquement entre les États de l'UE. L'échange sera fondé sur les règles existantes de la directive 2011/16/UE relatives aux modalités pratiques de l'échange d'informations, y compris l'utilisation de formulaires types.

Ces informations permettront aux autorités fiscales de réagir aux pratiques fiscales dommageables grâce à des modifications de la législation, ou encore par des évaluations des risques et des contrôles fiscaux adéquats. Une transparence accrue devrait également inciter les groupes d'entreprises multinationales à abandonner certaines pratiques fiscales hasardeuses et à payer leur juste part d'impôt dans les États où ils réalisent leurs bénéfices.

Ailleurs qu'au sein de l'UE, plusieurs États et territoires, dont le Canada, ont signé l'Accord multilatéral entre autorités compétentes concernant l'échange de déclarations pays par pays. Cet accord est le fruit des travaux du plan d'action BEPS (Base Erosion and Profit Shifting - érosion de la base d'imposition et transfert de bénéfices) entrepris au lendemain de la crise de 2008-2009 par l'OCDE en collaboration avec le G20.

De la même manière que ce qui est prescrit dans l'UE, la déclaration pays par pays exigera, de la part de toutes les entreprises multinationales dont le revenu annuel consolidé dépasse 750 millions d'euros (environ 1,1 milliard de dollars canadiens), qu'elles produisent annuellement une déclaration pays par pays indiquant le chiffre d'affaires consolidé, les bénéfices, l'impôt payé ou dû, le nombre d'employés, le capital déclaré, les bénéfices non distribués, les actifs corporels et ce, pour chaque juridiction où elles exercent 
une activité. Ces renseignements seront ensuite partagés automatiquement avec les administrations fiscales des autres signataires de l'Accord, qui pourront ainsi dresser un tableau plus complet et unifié des indicateurs clés de l'activité des entreprises multinationales ayant une activité sur leur territoire. L'obligation de transparence accrue de la part des entreprises multinationales permettra, ultimement, aux administrations fiscales de s'assurer qu'elles paient leur juste part d'impôt dans les États où leurs profits sont générés. Les premiers échanges ont commencé en 2017.

Mais les obligations de transparence ne viendront pas seulement des législateurs ou d'autorités supranationales, comme l'UE. C'est ainsi que les normes de publication d'informations financières doivent conduire les entreprises à se soucier davantage du risque lié à leur comportement fiscal international. Car l'intensification de l'obligation de transparence à propos des comportements fiscaux de l'entreprise connait déjà une traduction sur le plan des normes comptables. Il s'agit de la norme FASB (Financial Accounting Standards Board) FIN 48 pour les États-Unis et le Canada, et de la norme IFRS (International Financial Reporting Standards) IAS 12 pour l'UE. En vertu de celle-ci, l'entreprise doit comptabiliser ses " positions fiscales incertaines », soit les risques fiscaux auxquels elle est exposée, dont ceux pouvant découler d'une remise en question, par les administrations fiscales, de certaines de ses pratiques fiscales internationales qu'elles considéreraient comme étant abusives. Cela entraîne pour elle l'obligation d'abord d'informer les administrations fiscales de tous les faits pertinents entourant ces opérations, puis d'établir des provisions correspondantes s'il est plus que probable qu'elles n'accepteront pas les positions en question.

Il résulte de ces nouvelles exigences de transparence imposées aux entreprises, dont l'emprise est vouée à se resserrer, que les stratégies fiscales agressives font aujourd'hui peser un risque multiforme avéré sur les entreprises qui y ont recours.

D'abord, les possibilités qu'elles soient décelées par les administrations fiscales sont aujourd'hui plus grandes, compte tenu de l'accroissement, d'une part, de l'effectif des administrations fiscales, comme le Canada y a procédé récemment et, d'autre part, des échanges de renseignements entre administrations, en voie de devenir bientôt automatique sur le plan mondial. Puis, en cas de découverte, un redressement aux conséquences fiscales dissuasives est susceptible d'être opéré par les administrations des États qui en subissent un préjudice. Ils y procéderont à l'aide des moyens législatifs fortifiés, quoique bien imparfaits, dont ils se sont dotés ces dernières années, et dont les lacunes seront corrigées par les États de l'OCDE et du G20 au fur et à mesure de l'avancement des travaux de la première, en collaboration avec la seconde, portant sur le plan d'action BEPS.

De toute façon, même si les pratiques de planification fiscale abusive ne sont pas détectées par les administrations fiscales, elles risquent néanmoins de l'être par les ONG. Celles-ci ne vont pas manquer, à l'avenir, de cibler les entreprises qui se livrent à de telles pratiques, de la même manière qu'elles le font pour celles qui sont adeptes de la corruption internationale. L'Internet et les réseaux sociaux électroniques aidant, le risque de réputation ou d'image s'accroît et devient, de ce fait, en passe de se réaliser. Peuvent ainsi s'ensuivre des campagnes de boycottage des produits ou services des entreprises au comportement fiscal contraire à celui d'un bon citoyen, avec des répercussions sur leurs résultats financiers, prélude à la baisse du cours des titres de celles qui sont cotées à une bourse de valeurs.

L'utilisation qui, autrefois, était faite des ETNC, et plus généralement des paradis fiscaux, ne sera ainsi plus la même à la suite des réformes qui ont pris naissance dans le sillage de la crise financière et économique de 2008-2009. Les États du G20, spécialement les plus développés d'entre eux, de même que l'OCDE, ont en effet affiché clairement leur volonté de mettre fin aux pratiques fiscales abusives autant des individus que des entreprises, qui les utilisent soit pour dissimuler des revenus, s'agissant surtout des premiers, soit afin d'y transférer des bénéfices, à propos spécialement des secondes. La «traque » de ces États et territoires avait déjà commencé à la fin des années 1990, principalement au sein de l'OCDE et de l'UE; mais le mouvement s'est, depuis, accéléré. Même s'il est difficile de prévoir jusqu'où ira le chambardement amorcé, il demeure que rien ne sera plus comme avant : la "moralisation » fiscale qui est en cours est susceptible de revêtir des proportions qui n'auraient pas été envisageables il y a seulement quelques années. Il aura fallu, pour cela, que survienne une crise majeure à la fin de la première décennie du nouveau millénaire, qui a laissé des États surendettés. Preuve, encore une fois, que le monde et les différents systèmes juridiques qui le composent et le structurent 
progressent normalement à petits pas, mais en accéléré dans la foulée de grands bouleversements. L'arrivée de Donald Trump à la présidence des États-Unis d'Amérique provoque cependant une immense incertitude quant à la poursuite du mouvement amorcé.

Les contraintes pesant sur les entreprises se livrant à l'optimisation fiscale abusive sont par conséquent devenues trop lourdes et clairement préjudiciables à leurs intérêts et à ceux de leurs actionnaires ${ }^{32}$. Non seulement leur comportement fiscal irresponsable n'est clairement plus de mise, mais encore, l'adoption de pratiques fiscales internationales vertueuses de leur part est devenue une nécessité. Certaines l'ont déjà compris.

Sentant le resserrement inéluctable des contraintes pesant sur elles, en particulier sur le plan de la transparence de leurs opérations internationales, les grandes entreprises œuvrant dans le domaine des TIC sont aujourd'hui disposées à adopter une attitude plus responsable sur le plan fiscal. Des tractations en ce sens auraient été, semble-t-il, engagées avec le gouvernement américain en 2013, dont le résultat n'a cependant pas filtré. Mais ces entreprises voulaient monnayer leur acte de contrition fiscal en échange d'une diminution du taux d'imposition appliqué à l'égard des bénéfices générés en dehors des États-Unis ${ }^{33}$. Leurs chances serontcependant meilleures avec le nouveau président Trump, car une telle mesure figurait dans le programme électoral qui fondé son élection. C’est ainsi qu'il désire inciter les multinationales américaines à rapatrier les 2500 milliards de dollars qu'elles conservent à l'étranger dans le but d'échapper à l'impôt américain. Il était disposé, pour cela, à leur accorder un cadeau fiscal en n'imposant ces sommes qu'à hauteur de $10 \%$ plutôt qu'au taux courant de $35 \%$. La grande réforme fiscale qu'il a présentée le 27 septembre 2017 fait toutefois silence sur cette question, quoique, par sa proposition de ramener à $20 \%$ le taux de l'impôt sur les sociétés, il pense sans doute atteindre le même objectif de rapatriement des sommes mises à l'abri sous des cieux fiscaux plus cléments.

Les nombreux appels à la discipline fiscale, qu'ils proviennent des législateurs ou des voix qui commandent l'application résolue de la RSE en raison des nombreux risques associés au fait de ne pas s'y conformer, ont été entendus, mais de façon curative seulement, par Starbucks. Devant les menaces de boycottage de ses produits et implantations en Europe agitées par certaines ONG, en particulier au Royaume-Uni, la société américaine a en effet annoncé, le 6 décembre 2012, qu'elle allait renoncer à ses pratiques d'optimisation fiscale et payer environ 20 millions de livres supplémentaires d'impôts en 2013 et 2014 (10 en 2013 et 10 en 2014$)^{34}$. Starbucks s'est par conséquent résolue à acquitter, que ses résultats fussent bénéficiaires ou non, un montant d'impôt supérieur à celui prévu par la loi. Pour ce faire, elle a renoncé, pour les deux années en question, d'abord à bénéficier des exemptions liées, notamment, au paiement de redevances pour l'utilisation de sa marque, puis à transférer des revenus vers des filiales implantées dans des États ou territoires à fiscalité privilégiée, pratiques qu'elle utilisait jusqu'alors pour diminuer sa charge fiscale totale en Europe.

Il en va de même de l'annonce faite par Amazon de payer ses impôts en Europe dans les États où elle réalise ses bénéfices. En 2013, l'agence Reuters avait en effet révélé qu'Amazon, en 2012, en faisant transiter ses ventes par sa filiale luxembourgeoise, n'avait payé en Allemagne que trois millions d'euros d'impôt sur les sociétés, alors que son chiffre d'affaires dans cet État avoisinait les neuf milliards d'euros. Le géant américain de la distribution de produits en ligne a ainsi annoncé, fin mai 2015, qu'il avait commencé à acquitter en Allemagne, et non plus au Luxembourg, ses impôts sur les bénéfices qu'il réalise sur le sol allemand, modification ayant pris effet le 1 er mai précédent ${ }^{35}$. Il faut dire que l'Allemagne est, hors des États-Unis d'Amérique, le plus important marché d'Amazon, car elle y réalise $12 \%$ de ses ventes mondiales et un tiers de ses ventes non réalisées en sol américain.

Assiste-t-on à l'amorce d'un retournement de situation qui entraînera, dans le mouvement, les autres entreprises se trouvant dans la même situation? S'agit-il d'une reconnaissance du risque de réputation ou d'image que peut entraîner la révélation de pratiques fiscales internationales abusives? L'hostilité désormais affichée par les citoyens à l'égard des stratégies fiscales de ces grands groupes, jusqu'ici sporadique mais que l'on sent grandir et dont les ONG ainsi que, désormais, les dirigeants politiques, se font l'écho, serait ressentie comme une menace par les entreprises concernées. C'est en particulier le cas de celles dont la valeur repose pour partie sur leur image, qui représente pour elles un actif stratégique de la plus haute importance, gage de leur survie. Ce repentir, bienvenu pour la santé financière des Trésors publics, est-il finalement 
une bonne chose si on l'envisage dans une perspective plus large, « citoyenne »?

La commission des Finances, de l'Économie générale et du Contrôle budgétaire de l'Assemblée nationale française a, à cet égard, émis un commentaire judicieux à propos du comportement d'entreprises telles que Starbucks et Amazon, qui, pour protéger leur réputation et, du coup, leur image de marque, décident spontanément d'adopter un comportement vertueux sur le plan fiscal. Elle s'est demandé, à bon droit, s'il s'agit là d'une bonne nouvelle pour la notion même d'impôt. Celui-ci, en effet, consiste en une contribution obligatoirement due par ceux qui en sont redevables. En démocratie, le consentement à l'impôt se manifeste par le vote des représentants des citoyens à l'Assemblée législative, tel que le requiert, en France, l'article 14 de la Déclaration des droits de l'homme et du citoyen de 1789. Il est à cet égard curieux, selon la commission, que des entreprises décident d'acquitter de manière discrétionnaire un supplément d'impôt dont elles fixent arbitrairement le montant, alors qu'elles n'en seraient pas juridiquement redevables. Il s'agit, à son avis, d'une forme nouvelle et déviante de consentement à l'impôt, à la manière d'une aumône que les entreprises accepteraient de verser. L'acceptation d'un tel comportement représenterait, de la part des États, un aveu de faiblesse, la consécration de l'idée que les plus fortunés peuvent décider à leur guise s'ils entendent contribuer aux charges fiscales, et, qui plus est, à quelle hauteur ${ }^{36}$.

Quoi qu'il en soit, la discipline fiscale, traduction d'un devoir social de même que moral de la part des entreprises, doit dès aujourd'hui figurer impérativement au cœur de leurs pratiques de RSE. L'autoréglementation n'est cependant qu'un prélude à une intervention inéluctable et prochaine, qui a même déjà commencé dans certains États, des législateurs nationaux (et des organes de l'UE) en vue d'instaurer un « civisme fiscal », devenant dès lors contraint.

Toutes ces initiatives, qu'elles proviennent des législateurs, des organisations internationales supranationales telle l'UE ou des entreprises elles-mêmes, ne feront sans doute pas disparaitre, quel que soit l'horizon que l'on puisse contempler, les États ou territoires à fiscalité privilégiée. Un grand pas aura pourtant été franchi. Il aura suffi d'une volonté politique commune de la part des chefs d'État et de gouvernement des États développés, et, plus largement, de ceux du
G20. Celle-ci, avec l'aide de l'OCDE, a permis l'adoption de dispositions obligatoires résolument appliquées, traduction des déclarations d'intention habituellement formulées, lors de la clôture des sommets du G20 tenus depuis 2008, à propos de la fin prochaine des paradis fiscaux. En ce sens, la question qui se pose aujourd'hui n'est plus de savoir si cette volonté adviendra, car elle existe déjà; il s'agit plutôt d'en mesurer la portée.

La coopération fiscale renforcée en matière d'échange de renseignements résultant des scandales fiscaux qui ont émaillé la dernière décennie a déjà grandement rehaussé les moyens d'identification des contribuables, personnes physiques, qui se livrent à des pratiques de fraude et d'évasion fiscales internationales. Le défi actuel dans cette avancée consiste à fortifier davantage l'échange de renseignements, mais cette fois à propos des pratiques fiscales des entreprises. L'instauration de celui-ci, désormais revêtu d'un caractère automatique, n'est plus qu'une question de mois entre la plupart des États ou territoires de la planète.

Les entreprises sont prévenues. À elles d'ajuster en conséquence leur comportement fiscal international pour que, dorénavant, il soit socialement responsable. Il en va de même à propos des pratiques constitutives de corruption internationale auxquelles elles pourraient être tentées de recourir. L'introduction ou le raffermissement de normes prescrivant la transparence des activités et pratiques fiscales internationales des entreprises ont déjà été réalisées, ou alors sont en voie de l'être, ce point étant inscrit au programme de travail des législateurs. Ces nouvelles obligations de divulgation bénéficieront non seulement aux autorités fiscales mais également aux actionnaires, pour les entreprises cotées à tout le moins, ainsi qu'aux ONG. Les informations sur leurs pratiques fiscales internationales étant ainsi rendues publiques, l'on voit tout l'intérêt pour les entreprises de devenir socialement responsables sur ce plan. Elles pourraient, à cet égard, chercher à obtenir un label de la part d'une ONG s'intéressant à la question, comme cela se fait déjà en matière de corruption internationale, afin de certifier, à tout le moins, qu'elles ne disposent pas d'établissements ou de filiales dans des ETNC.

Car les risques découlant d'un comportement fiscal international non responsable de la part des entreprises, évoqués précédemment, sont nombreux. Ils 
sont d'ailleurs destinés à épouser des formes nouvelles, comme l'exclusion de l'attribution de contrats ou d'aides par certaines autorités publiques, comme il en va déjà en matière de corruption. C'est ce qu'ont préconisé, parmi d'autres mesures portant en particulier sur la transparence des opérations des entreprises multinationales, les députés siégeant au Parlement européen, dans une résolution adoptée lors d'une session plénière tenue le 8 juillet 2015. La résolution va en effet dans le sens qu'aussi bien les institutions financières de l'UE, comme la Banque européenne d'investissement, que celles qui, au sein des États membres, financent le développement, devraient exercer une surveillance sur les entreprises qui reçoivent leur aide afin de veiller à ce qu'elles n'aient pas recours à l'optimisation fiscale internationale abusive. La connexité entre les deux types de comportement répréhensible des entreprises sur lesquels le présent article s'est concentré devient ici on ne peut plus évidente. Les mandataires sociaux des entreprises ayant une activité internationale significative ont donc tout intérêt à considérer la discipline fiscale, en particulier dans son volet international, comme partie intégrante d'une bonne gouvernance d'entreprise.

\section{Conclusion}

Les pratiques de corruption et de fiscalité abusive forment les deux types de comportement déviant des entreprises sur le plan international qui marquent le plus les esprits en raison de leur évocation régulière dans les médias. Ce ne sont toutefois pas les seules. Le transfert international de leur siège social pourrait aussi, en fonction des motifs qui y président, figurer au nombre. Mais, plus largement, toutes ces pratiques, en dépit de leur légalité apparente et même réelle dans plusieurs cas, et justement à cause d'elle parce qu'elles se situent à sa marge, posent des interrogations à propos de leur légitimité. Les questions de légitimité conduisent, par ricochet, à des interrogations qui se situent sur un plan proprement éthique. Car il ne suffit pas de respecter les législations nationales. S'en tenir à cette seule considération reviendrait à masquer les véritables enjeux. Certes, ceux-ci comportent des aspects juridiques indéniables; mais, comme les comportements répréhensibles identifiés déploient leurs répercussions sur les plans économique et social, la perspective doit s'élargir afin d'embrasser une optique extrajuridique. C'est pourquoi la question de la RSE, qu'elle soit spontanée ou contrainte, a été évoquée dans le cadre du présent article.
Les entreprises sont les piliers de l'économie de marché. Sans elles, il n'y a pas de système économique qui vaille. Elles sont tenues, bien évidemment, de ne pas enfreindre les législations étatiques. Mais leur responsabilité va bien au-delà, quoi qu'on en dise. Bien que leur création et leur développement doivent être favorisés, il n'en demeure pas moins que leur statut privilégié sur les plans juridique (en particulier la personnalité morale dont elles sont habituellement revêtues) et fiscal (sur ce plan, en particulier un taux d'imposition moindre que celui s'appliquant aux individus) leur confère des responsabilités non seulement à l'égard de leurs actionnaires, mais aussi à l'endroit de toutes les autres parties prenantes, directes et indirectes.

Bien sûr, certaines législations peuvent les autoriser à octroyer des paiements de facilitation aux agents publics étrangers. De même peuvent-elles, lorsqu'il n'y a pas de disposition législative ou conventionnelle contraire, faire jouer les lacunes et asymétries provenant de l'interaction des législations fiscales, afin de ne payer qu'un minimum d'impôt, voire aucun impôt, dans les États où elles réalisent leurs bénéfices. Il s'agit pourtant de comportements qui n'apparaissent plus acceptables dans la plupart des cas, car ils sont entachés d'abus ou effleurent celui-ci. Ils entraînent en outre un report de la charge fiscale éludée sur les épaules des contribuables, personnes physiques, qui, eux, ne peuvent y échapper.

Le droit de la plupart des États développés consacre cette notion d'abus de droit. Elle est toutefois difficile à appliquer. Les entreprises, surtout celles qui disposent des moyens humains et financiers les plus importants, peuvent ordinairement, ne serait-ce qu'au bout d'un long processus, obtenir gain de cause contre les États qui s'attaquent à elles sur ce fondement. Encore ne s'agirait-il là que d'une victoire à la Pyrrhus. Car peut-on parler d'un vrai succès si celui qui le remporte l'obtient aux dépens de ceux (la société au sens large) qui lui accordent d'immenses privilèges dont euxmêmes ne bénéficient pas?

De leur côté, l'OCDE et les États du G20, autant individuellement que collectivement, depuis la crise de 2008-2009, tentent, par les moyens dont ils disposent, de couper court aux manœuvres des entreprises qui sont considérées comme n'étant plus acceptables ou tolérables aujourd'hui, la corruption et les pratiques fiscales abusives dans les opérations internationales se situant au premier rang. Ils pénalisent, d'une part, les 
entreprises qui se livrent à ce genre d'activités; ils appliquent, d'autre part, une dense pression sur les États ou territoires où, généralement, ces pratiques répréhensibles se déroulent, afin qu'ils modifient leur législation et ensuite procèdent à leur application (en ce qui concerne la corruption), ou bien qu'ils échangent des renseignements fiscaux et financiers (en ce qui touche les pratiques fiscales abusives).

Certains États ou territoires autrefois récalcitrants commencent à le comprendre, sentant probablement que l'étau qui se resserre sur eux depuis 2009 ne relâchera pas sa pression, bien au contraire. Ainsi, le 14 octobre 2014, monsieur Michael Noonan, le ministre irlandais des Finances, annonçait, pour 2020, la fin du double Irish. Il s'agit d'une technique jusque-là permise d'optimisation fiscale très controversée, utilisée par les grandes multinationales américaines, dont Google, qui l'emploie dans le but de délocaliser ses bénéfices aux Bermudes, paradis fiscal classique, en les faisant transiter par une société hybride de droit irlandais ${ }^{37}$.

Le combat est pourtant loin d'être gagné. Il faut d'abord que tous les États et territoires coopèrent, car s'il s'en trouvait un seul ne jouant pas suivant les règles, sur le plan des pratiques fiscales à tout le moins, l'action résolue enclenchée depuis la fin de 2008 sur les deux fronts de la pénalisation et de l'encerclement, en serait détruite ${ }^{38}$. Il y aura toujours, par ailleurs, des entreprises à même de déceler les échappatoires, qu'elles se situent dans les législations nationales ou au sein des conventions fiscales, leur permettant de poursuivre leurs pratiques internationales de corruption et de fiscalité abusive, et ce en dépit des résultats des travaux engagés par l'OCDE dans le cadre du plan d'action BEPS, qui visent justement à mettre fin aux secondes.

Les entreprises, en particulier celles ayant une activité internationale importante, doivent pourtant prendre conscience de ce constat implacable : elles sont de plus en plus cernées par le droit ou par des moyens parajuridiques. Les deux types de comportement sur lesquels le présent article a mis l'accent et qui ont lieu dans le cadre de leurs opérations internationales, ne sont plus considérés comme acceptables par les États du G20, de même que par l'OCDE. Les contraintes vont inéluctablement se refermer sur celles qui se livrent encore à ce genre de pratique.

Il ne sert donc à rien pour elles de tergiverser : les entreprises n'ont d'autre choix que d'orienter leurs efforts dans le sens de la RSE, qui, de toute façon, leur sera imposée progressivement par les législateurs (et les organes de l'UE). L'adoption, qu'elle soit spontanée ou contrainte, d'un comportement vertueux, est ainsi vouée à devenir une condition obligée de leur viabilité à long terme. D’où la nécessité de faire figurer les deux comportements répréhensibles que sont la corruption internationale et les pratiques fiscales internationales abusives au sein de leur code de conduite, qui les interdira en toutes circonstances, et, afin d'assurer le respect de l'interdiction, prévoira des mécanismes de contrôle et de conformité (monitoring). Il n'y a pas d'autre issue possible; sinon, les conséquences pénales et fiscales, de même que celles à caractère commercial et financier, provenant de la réalisation du risque de réputation, sont trop lourdes pour qu'une entreprise ose s'y exposer. Il est préférable pour elles de le réaliser et de se mettre pleinement à la manœuvre de la RSE, non par un comportement de façade mais par une réelle adhésion, se traduisant par un code de conduite pleinement effectif, contrôlé et vérifié. Il revient à cet égard aux dirigeants et autres mandataires sociaux de donner l'exemple d'une gouvernance d'entreprise qui aille hardiment dans cette direction. Le temps de la RSE est désormais arrivé. Il revient aux entreprises d'agir en ce sens au plus vite, stimulées en cela par des dirigeants responsables et visionnaires.

\section{NOTES}

1 Certains éléments de cet article sont inspirés de la première des deux parties de l'ouvrage rédigé par l'auteur et publié en 2015 aux Éditions Berger-Levrault, dans la collection «Au fil du débat », et portant le titre Corruption et fiscalité - L'entreprise face à ses pratiques internationales.

2 OCDE (2014). Rapport de l'OCDE sur la corruption transnationale - Une analyse de l'infraction de corruption d'agents publics étrangers. Paris, France : OCDE. Repéré à http://www.oecd-ilibrary.org/docserver/download/2814012e.pdf?expires $=1507393424 \& \mathrm{id}=\mathrm{id} \&$ accname $=$ guest $\&$ checksum $=1$ CE5EEE789083E00BB9142EF9987242D 
3 Selon les calculs des auteurs, une augmentation du taux d'imposition de $1 \%$ conduit à une diminution du même pourcentage du taux de croissance de l'entreprise. Fisman, R, Svennson, J. (2007). Are corruption and taxation really harmful to growth? Firm level evidence. Journal of Development Economics, 83, 63-75.

4 Sentence Chambre de commerce internationale [CCI ou International Chamber of Commerce (ICC)], aff. 1110/1963.

5 Une clause compromissoire est une disposition incluse dans un contrat ou un traité en vertu de laquelle les parties s'entendent pour faire trancher par un tribunal arbitral tout litige futur portant sur l'interprétation ou l'application du contrat ou du traité.

6 Sentence CCI, aff. 3916/1982, JDI, 1984, p. 930.

7 Sentence CCI, aff. 3913/1981

8 CA Paris, 1re chambre civile, European Gas Turbines $S$ A c/Westman International Ltd., 10 septembre 1993, Revue critique de droit international privé, 1994, 83, 349-367, note V. Heuzé; Revue de l'arbitrage, 1994, 359-379, note D. Bureau.

9 Propos annexe, qui ne vient pas appuyer la motivation qui sert d'assise au dispositif (conclusion) d'une décision de justice ou d'une sentence arbitrale.

10 Peuvent aussi être mentionnées les sentences arbitrales suivantes, prononcées plus récemment : Inceysa V allisolentana S.L. $v$. Republic of El Salvador, Centre international pour le règlement des différends relatifs aux investissements (CIRDI), no ARB/03/26, 2 août 2006; World Duty Free Company Limited v. Republic of Kenya, CIRDI, no ARB/00/7, 4 octobre 2006.

11 Cette expression est aussi utilisée pour justifier les paiements de facilitation.

12 Lois du Canada (2013), chapitre 26.

13 C'est-à-dire non étatiques, mais dotés d'autonomie sur le plan fiscal. Il en va ainsi, par exemple, des îles de Jersey et Guernesey, ou encore des Bermudes.

14 Gendron, C. (2013). Les pratiques fiscales : une occasion de questionner le périmètre de la responsabilité sociale de l'entreprise. Environnement et développement durable, 2 (février), 19-26.

15 Propos de J. Stead, porte-parole de l'ONG Christian Aid, rapportés dans l'article de M. Roche, «Londres s'attaque à l'optimisation et à l'évasion fiscales ", Le Monde, 4 déc. 2012.

16 Les divers arguments invoqués de part et d'autre sont relevés par Gouthière, Br., Les impôts dans les affaires internationales, 14e éd., Paris, Francis Lefebvre, 2014, no 70555.

17 Voir Gutmann, D., «Les instruments non fiscaux de lutte contre l'évasion fiscale - Réflexions sur la responsabilité sociale des entreprises ", Revue française de finances publiques, no 110, avril 2010, p. 57-69; Droit fiscal des affaires, 3e éd., Paris, Montchrestien, coll. « Domat droit privé », 2012, nos 1033-1037.

18 International Organization for Standardization.

19 Quoique ce chapitre ne contienne que deux paragraphes, ceux-ci sont évocateurs, surtout en raison des commentaires qui les accompagnent, et qui figurent aux nos 100 à 106.

20 Sénat de France (2012). Rapport no 673 fait au nom de la commission d'enquête sur l'évasion des capitaux et des actifs hors de France et ses incidences fiscales. Repéré à https://www.senat.fr/rap/r11-673-1/r11-673-11.pdf

21 Assemblée nationale de France (2013). L’optimisation fiscale des entreprises dans un contexte international, rapport d'information (no 1243) déposé par la commission des Finances, de l'Économie générale et du Contrôle budgétaire, en conclusion des travaux d'une mission d'information, Repéré à http://www.assemblee-nationale.fr/14/pdf/rapinfo/i1243.pdf

22 Loi no 2001-420 du 15 mai 2001 relative aux nouvelles régulations économiques.

23 Loi no 2010-788 du 12 juillet 2010 portant engagement national pour l'environnement. Les dispositions en question sont aujourd'hui incluses dans le Code de commerce, à l'article L. 225-102-1. Seules sont concernées certaines entreprises, en fonction de seuils définis par le décret no 2012-557 du 24 avril 2012 relatif aux obligations de transparence des entreprises en matière sociale et environnementale.

24 Gutmann, D., op. cit., note 16, no 1037.

25 Assemblée nationale de France, op. cit., note 20, p. 122-123.

26 Article 7 de la loi.

27 Loi nº 2016-1691 du 9 décembre 2016 relative à la transparence, à la lutte contre la corruption et à la modernisation de la vie économique.

28 Décision no 2016-741 DC du 8 décembre 2016, nos 100 à 104 de la décision. 
$29 \mathrm{COM} / 2015 / 302 /$ final.

30 Directive (UE) 2015/2376 du Conseil du 8 décembre 2015 modifiant la directive 2011/16/UE en ce qui concerne l'échange automatique et obligatoire d'informations dans le domaine fiscal.

31 Directive (UE) 2016/881 du Conseil du 25 mai 2016 modifiant la directive 2011/16/UE en ce qui concerne l'échange automatique et obligatoire d'informations dans le domaine fiscal.

32 OCDE (2013). Lutter contre l'érosion de la base d'imposition et le transfert de bénéfices. Paris, France: OCDE, 2013, p. 35 (format PDF).

33 Cypel, S. (2013, 21 mai). Apple mis en cause pour ses détournements d'impôts. Le Monde.

34 S.n.a. (2012, 6 décembre). Starbucks cède et accepte de payer plus d’impôts au Royaume-Uni. lemonde.fr.

35 Eveno, A. (2015, 25 mai). Amazon commence à payer ses impôts là où il fait des bénéfices. Le Monde.

36 Assemblée nationale de France, op. cit., note 20, p. 124-128.

37 Albert, É. (2014, 17 octobre). Les grandes multinationales sereines après la fin du « double irish. Le Monde Économie.

38 Avi-Yonah, R.S. (2015). Advanced introduction to international tax law. Cheltenham, UK) / Northampton, MA: Edward Elgar, coll. «Elgar Advanced Introductions ». 